\title{
Fludarabine, mitoxantrone and dexamethasone as a treatment for refractory T-cell large granular lymphocyte leukemia
}

\author{
Jing Liu ${ }^{1}$, Lihua Dong ${ }^{1}$, peng Zu ${ }^{1}$, Jingjing Huang ${ }^{1}$, and Yufu $\mathrm{Li}^{1}$ \\ ${ }^{1}$ Henan Cancer Hospital
}

October 29, 2020

\begin{abstract}
T-cell large granular lymphocyte leukemia (T-LGLL) is a rare clonal lymphoproliferative disorder. Because of its low incidence rate and few cases, there is no standard treatment guideline. We report on a case of T-LGLL with severe anemia [hemoglobin (HGB): $36 \mathrm{~g} / \mathrm{L}]$. After 4 months of treatment with cyclosporine A, the response was suboptimal, and the patient still had transfusion-dependent anemia (HGB: $47 \mathrm{~g} / \mathrm{L}$ ). After six cycles of FND (fludarabine, mitoxantrone, dexamethasone) regimen, the hematological response was complete (HGB: $143 \mathrm{~g} / \mathrm{L}$ ) and the monoclonal lymphocytes were nonexistent. Therefore, an FND regimen may be an option for treating refractory T-LGLL.
\end{abstract}

Fludarabine, mitoxantrone and dexamethasone as a treatment for refractory T-cell large granular lymphocyte leukemia

Jing Liu ${ }^{1}$, Lihua Dong ${ }^{1}$, Peng $\mathrm{Zu}^{1}$, Jingjing Huang ${ }^{1}$, Yufu $\mathrm{Li}^{1 *}$

${ }^{1}$ Department of Hematology, Affiliated Cancer Hospital of Zhengzhou University, Zhengzhou, Henan 450008, China

$1^{*}$ Corresponding to: Yufu Li; Department of Hematology, Affiliated Cancer Hospital of Zhengzhou University, Zhengzhou, Henan 450008, China ,E-mail address:liyufu439@126.com

Text word counts: 1126

Abstract word counts: 97

Keywords: Large granular lymphocyte leukemia; Fludarabine;Treatment

Short running title: FND in T-LGLL

Tables: 1

Fgures: 1

Abbreviations:

\begin{tabular}{ll}
\hline T-LGLL & T-cell large granular lymphocyte leukemia \\
\hline FND & fludarabine mitoxantrone dexamethasone \\
HGB & hemoglobin \\
WBC & white blood cell \\
NEU & neutrophil \\
LYM & lymphocyte \\
PLT & platelet
\end{tabular}




\begin{tabular}{ll}
\hline T-LGLL & T-cell large granular lymphocyte leukemia \\
\hline FCM & low cytometry \\
CsA & cyclosporine A \\
MTX & methotrexate \\
Cy & cyclophosphamide \\
ORR & overall response rate \\
\hline
\end{tabular}

\begin{abstract}
T-cell large granular lymphocyte leukemia (T-LGLL) is a rare clonal lymphoproliferative disorder. Because of its low incidence rate and few cases, there is no standard treatment guideline. We report on a case of T-LGLL with severe anemia [hemoglobin (HGB): $36 \mathrm{~g} / \mathrm{L}$ ]. After 4 months of treatment with cyclosporine A, the response was suboptimal, and the patient still had transfusion-dependent anemia (HGB: $47 \mathrm{~g} / \mathrm{L}$ ). After six cycles of FND (fludarabine, mitoxantrone, dexamethasone) regimen, the hematological response was complete (HGB: $143 \mathrm{~g} / \mathrm{L}$ ) and the monoclonallymphocytes were nonexistent. Therefore, an FND regimen may be an option for treating refractory T-LGLL.
\end{abstract}

Keywords: Large granular lymphocyte leukemia; Fludarabine;Treatment

T-cell large granular lymphocytic leukemia (T-LGLL) is a kind of lymphoproliferative disease with an inert process. It is rare, accounting for $2 \%-3 \%$ of lymphocytic leukemia. Most T-LGLL occur in 45-75-yearold patients, and the male-to-female ratio is 1:1[1]. The clinical manifestations of the disease are anemia, neutropenia, repeated infections, and splenomegaly. Without clinical specificity, it is easy to miss and misdiagnose. Due to the small number of cases and the lack of systematic clinical research, there is no standard treatment. A case of T-LGLL treated successfully with FND (fludarabine, mitoxantrone, dexamethasone) is reported as follows.

A 34-year-old male presented to hospital on June 20, 2018, with 'dizziness and fatigue for nearly 3 months'. There was no obvious cause for the dizziness, fatigue, sweating, and other discomforts during the previous 3 months. He did not have palpable peripheral lymphadenopathy, splenomegaly, or hepatomegaly. The laboratory workup revealed the following: white blood cell (WBC) count:13.46 × 10 $/ \mathrm{L}$, neutrophil (NEU) count: $1.08 \times 10^{9} / \mathrm{L}$, lymphocyte $(\mathrm{LYM})$ count: $11.75 \times 10^{9} / \mathrm{L}$, HGB: $36 \mathrm{~g} / \mathrm{L}$, platelet $(\mathrm{PLT})$ count: $260 \times 10^{9}$ /L. Hepatitis B surface antibody, anti-hepatitis C virus, anti-human immunodeficiency virus, cytomegalovirus, and Epstein-Barr virus were negative, as was the antinuclear antibody spectrum. Direct and indirect Coombs tests were negative. Liver and kidney function, thyroid function, and immunoglobulin were not significantly abnormal. VitB12 and ferritin were normal. Peripheral blood smears showed that the proportion of lymphocytes were significantly increased, and granules could be seen in most of their cytoplasm (Figure 1 ). Bone marrow smears showed that erythroid proliferation was active, and mature erythroblasts were slightly different in size. The percentage of lymphocytes was normal, and a large number of granules were observed. Bone marrow flow cytometry (FCM) showed an abnormal mature T lymphocyte group, accounting for about $91.53 \%$ of the lymphocytes, with a phenotype of TCR $\alpha \beta^{+}, \mathrm{CD} 8^{+}, \mathrm{CD} 5^{+}, \mathrm{CD} 3^{+}, \mathrm{CD} 2^{+}, \mathrm{CD} 57^{-}$ , CD $56^{-}, \mathrm{CD}^{-}, \mathrm{CD} 30^{-}, \mathrm{HLA}-\mathrm{DR}{ }^{-}, \mathrm{CD} 38^{-}, \mathrm{CD} 20^{-}, \mathrm{TCR} \beta / \gamma^{-}$. TCRV $\beta$ repertoire analysis showed that V $\beta 3$ accounted for $81.05 \%$ of the lymphocytes and was considered to be a monoclonal lymphocyte cell. The STAT3 gene was negative for mutations, TCR gene rearrangement was positive, and no obvious abnormality was found from the bone marrow biopsy. Chromosome: 46, XY [20]. No abnormality was found in 41 genes related to myelodysplastic syndrome. Therefore, T-LGLL was diagnosed.

During hospitalization, the patient was initially treated with cyclosporine A (CsA) $100 \mathrm{mg}$ once every 12 $\mathrm{h}$ for 4 months. However, the response was suboptimal and the patient was still transfusion dependent. Routine blood showed the following: WBC count: $11.53 \times 10^{9} / \mathrm{L}$, NEU count: $1.28 \times 10^{9} / \mathrm{L}$, LYM count: $9.88 \times 10^{9} / \mathrm{L}, \mathrm{HGB}: 47 \mathrm{~g} / \mathrm{L}$, PLT count: $270 \times 10^{9} / \mathrm{L}$. Bone marrow FCM demonstrated persistent LGLs, with $86.02 \%$ involvement in lymphocytes. Considering the poor effect of CsA and the patient not tolerating the severe anemia, an FND regimen was given, which consisted specifically of the following: fludarabine $\left(25 \mathrm{mg} / \mathrm{m}^{2} / \mathrm{d} \mathrm{d} 1-\mathrm{d} 3\right)$, mitoxantrone $\left(10 \mathrm{mg} / \mathrm{m}^{2} \mathrm{~d} 1\right)$, and dexamethasone $(20 \mathrm{mg} / \mathrm{d} \mathrm{d} 1-\mathrm{d} 5)$, repeated every 4 
weeks for six courses (Table 1 ). After the second cycle, HGB was $71 \mathrm{~g} / \mathrm{L}$ and LGLs decreased to $60.37 \%$ of the lymphocytes by bone marrow FCM, part of the hematological reaction was obtained. After the fourth cycle, HGB was $123 \mathrm{~g} / \mathrm{L}$, bone marrow FCM showed that LGLs about $25.96 \%$ of the lymphocytes. The patient obtained a complete hematological response. More important, the abnormal monoclonal cells were significantly reduced compared with before. And after the sixth cycle, all the hematological parameters were in the normal range, no monoclonal cells were found by bone marrow FCM.TCR gene rearrangement was negative. The patient experienced no adverse reactions except for mild nausea and transient mild leukopenia during the treatment. At 18 months follow-up, the patient remained in complete remission without any other therapy.

T-LGLL is a rare lymphoproliferative disorder that is caused by sustained immune stimulation and dysregulation of apoptosis [2]. Immunosuppressive agents such as methotrexate (MTX), cyclophosphamide (Cy), and CsA are the main treatments for T-LGLL. The three latest retrospective studies show the overall response rate (ORR) of the three immunosuppressants are nearly $47 \%-53 \%$ [3-5]. Although the ORRs are similar, the ORR with Cy is higher than CsA and MTX. Cy as a second-line treatment after failure of MTX treatment has a certain effect. Therefore, Cy may be a more effective first-line treatment for T-LGLL[3, 4]. However, the complete response rate of these immunosuppressants is limited, recurrence is common, and the long-term medication will lead to infection and an increased risk of cancer. Thus, a more effective and persistent treatment is needed. Alemtuzumab, a monoclonal antibody against CD52, has shown certain efficacy. A phase 2 study shows that the remission rate is about $67 \%$ in 21 patients with T-LGLL [6]. Splenectomy can be used for splenomegaly with hemocytopenia, the total response rate is $58 \%$ [3]. In addition, it is reported that calcitriol can reduce the activation of STAT1 and STAT3 and the output of inflammatory cytokines, so the vitamin D supplementation may be a feasible treatment option for T-LGLL[7]. A STAT3 inhibitor is also promising. Fludarabine has also shown good efficacy with the potential to clear LGL cell clones. Costa et al.[8] reports that all six T-LGLL patients who received fludarabine treatment has a hematological response, while five cases (83.3\%) achieve absence of monoclonal populations as assessed by PCR. The adverse reactions are tolerable. In addition, it is reported that three patients with T-LGLL receive a complete hematological response after first-line treatment with the FND regimen [9]. Fludarabine as a cytotoxic drug not only can reduce the tumor cells but also can modulate distinct functions of the immune system to improve post-treatment tumor control [10].So we chose the FND regimen as the second-line treatment for this patient, who achieved complete hematological response and an eradication of LGL clones (Table 1 ).

In conclusion, FND has rapid response and good compliance, and may lead to persistent relief by eradicating the LGL clones. it may be a feasible alternative therapy for refractory T-LGLL and more particularly in patients who cannot tolerate long-term use of immunosuppressants. But it still needs further clinical research.

\section{Acknowledgments}

We thank the patient and written informed consent for publication of their details was obtained from the patient.

\section{Disclosure statement}

All authors have stated that they have no conflict of interest.

\section{References}

1. O'Malley D: T-cell large granular leukemia and related proliferations . Am J Clin Pathol 2007, 127(6) :850-859.

2. Lamy T, Loughran TP, Jr.: How I treat LGL leukemia .Blood 2011, 117 (10):2764-2774.

3. Sanikommu SR, Clemente MJ, Chomczynski P, Afable MG, 2nd, Jerez A, Thota S, Patel B, Hirsch C, Nazha A, Desamito J et al :Clinical features and treatment outcomes in large granular lymphocytic leukemia (LGLL) . Leuk Lymphoma 2018,59 (2):416-422. 
4. Zhu Y, Gao Q, Hu J, Liu X, Guan D, Zhang F: Clinical features and treatment outcomes in patients with T-cell large granular lymphocytic leukemia: A single-institution experience . Leuk Res2020, $90: 106299$.

5. Braunstein Z, Mishra A, Staub A, Freud AG, Porcu P, Brammer JE:Clinical outcomes in T-cell large granular lymphocytic leukaemia: prognostic factors and treatment response $\mathrm{Br} J$ Haematol 2020 .

6. Dumitriu B, Ito S, Feng X, Stephens N, Yunce M, Kajigaya S, Melenhorst JJ, Rios O, Scheinberg P, Chinian $\mathrm{F}$ et al :Alemtuzumab in T-cell large granular lymphocytic leukaemia: interim results from a single-arm, open-label, phase 2 study . The Lancet Haematology 2016, 3 (1):e22-e29.

7. Olson KK, PM; Olson, TL; et al.: Vitamin D decreases STAT phosphorylation and inflammatory cytokine output in T-LGL leukemia .Cancer Biol Ther 2017, 18(5) :290-303.

8. Costa RO, Bellesso M, Chamone DAF, Ruiz MA, Hallack Neto AE, Aldred VL, Pereira J: T-cell large granular lymphocytic leukemia: treatment experience with fludarabine . Clinics 2012,67 (7):745748 .

9. Tse E, Chan JC, Pang A, Au WY, Leung AY, Lam CC, Kwong YL:Fludarabine, mitoxantrone and dexamethasone as first-line treatment for T-cell large granular lymphocyte leukemia .Leukemia 2007, 21 (10):2225-2226.

10. Gassner FJ, Weiss L, Geisberger R, Hofbauer JP, Egle A, Hartmann TN, Greil R, Tinhofer I: Fludarabine modulates composition and function of the $T$ cell pool in patients with chronic lymphocytic leukaemia . Cancer Immunol Immunother 2011, 60 (1):75-85.

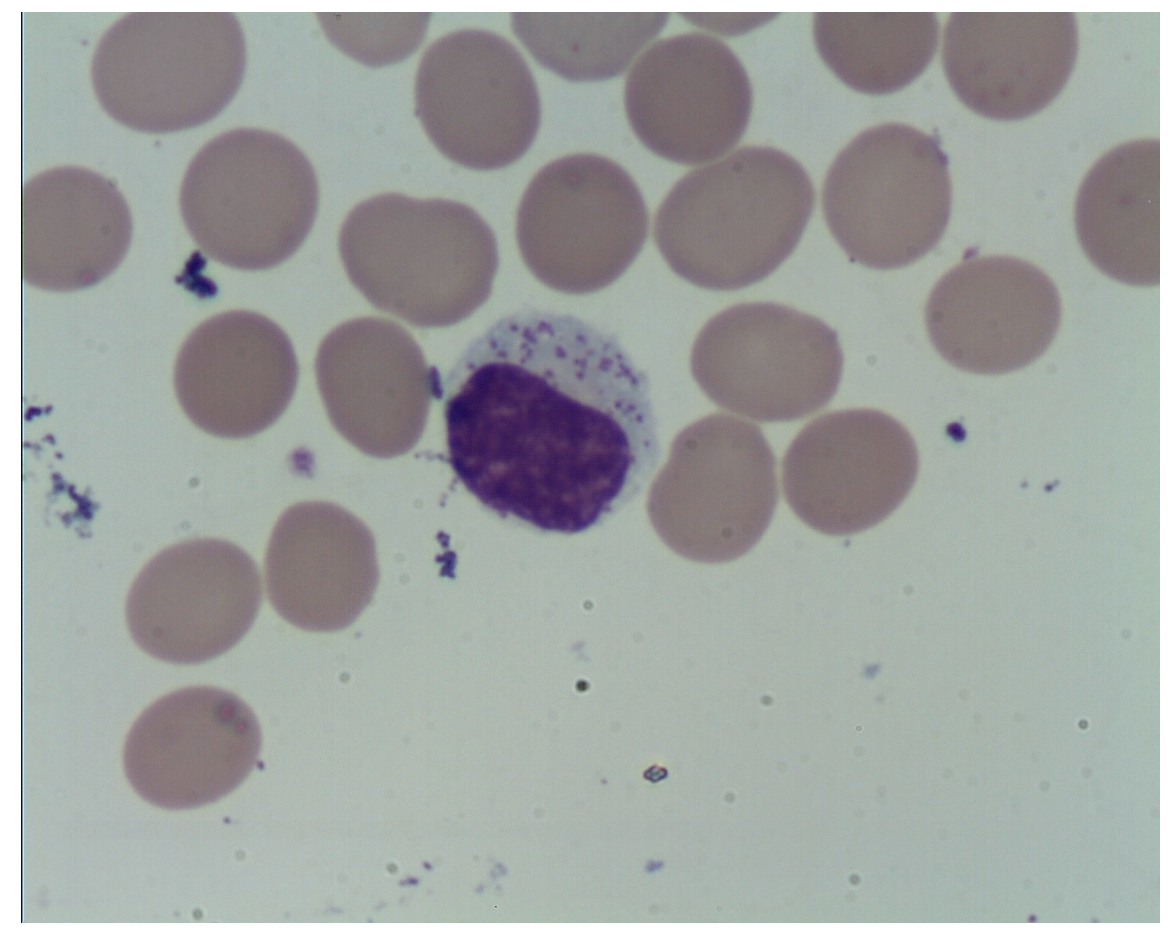

\section{Hosted file}

Table 1.pdf available at https://authorea.com/users/371232/articles/489651-fludarabinemitoxantrone-and-dexamethasone-as-a-treatment-for-refractory-t-cell-large-granular- 
lymphocyte-leukemia 